\title{
IoSiS - a Radar System for Imaging of Satellites in Space
}

\author{
M. Jirousek*, S. Anger, S. Dill, E. Schreiber, M. Peichl \\ German Aerospace Center, Muenchener Str. 20, 82230 Wessling, Germany, +49-8153-28-3506
}

\begin{abstract}
Space debris nowadays is one of the main threats for satellite systems especially in low earth orbit (LEO). More than 700,000 debris objects having potential to destroy or damage a satellite are estimated. The effects of an impact often are not identifiable directly from ground. Hence highresolution radar images are an appropriate tool for analyzing satellite structures. Therefor DLR is currently developing a radar system called IoSiS (Imaging of Satellites in Space), being presently based on an existing steering antenna facility and our multi-purpose high-performance radar system GigaRad [3], developed and constructed for multi-purpose experimental investigations. GigaRad is a multi-channel system operating at X band and using a bandwidth of up to $4.4 \mathrm{GHz}$ in the IoSiS configuration, providing fully separated transmit (TX) and receive (RX) channels, and separated antennas. For the observation of small satellites or space debris a high-power traveling-wave-tube amplifier (TWTA) is mounted close to the TX antenna feed. For the experimental phase IoSiS uses a $9 \mathrm{~m} T X$ and a $1 \mathrm{~m} R X$ antenna mounted on a common steerable positioner. High-resolution radar images are obtained by using Inverse Synthetic Aperture Radar (ISAR) techniques. The guided tracking of known objects during overpass allows wide azimuth observation angles. Thus high azimuth resolution comparable to the range resolution can be achieved. This paper outlines technical main characteristics of the IoSiS radar system including the basic setup of the antenna, the radar instrument and the corresponding RF error correction methodology, and the measurement strategy. Also a short description about a simulation tool for characterizing the whole instrument and exploring the expected nature of the radar images are discussed.
\end{abstract}

Keywords: ISAR, inverse SAR, space situation awareness, space debris, high resolution radar, radar sensor

\section{INTRODUCTION}

Currently there exist more than 500 operational satellites in a LEO. Main application areas of such satellite systems are communication, remote sensing, and military reconnaissance and surveillance. The high-end solution in complexity of course is given by the International Space Station (ISS). The wide range of applications and the resulting dependence of industrialized nations on such space systems cause the fact, that possible failures can have serious consequences. In order to verify unexplainable changes of the system performance, such as mechanical malfunction of the platform or electronic failures, both being indicators of possible mechanical damage, a regular assessment of the situation should be performed. Furthermore the cost of typically several hundred million Euros per satellite and the increasing number of space debris demands for preventive protection, which can be supported by a continuous ground-based observation. In order to address some of those gaps the experimental system IoSiS is currently under final construction. IoSiS is based on broadband X-band radar allowing range resolution of a few centimeters. In combination with a steerable antenna system, wide azimuth angles and thus high azimuth resolution can be achieved. This enables the application of ISAR techniques for precise investigation of object details and hence the detection of possible mechanical damages. 


\section{SYSTEM CONCEPT}

\subsection{Imaging principle}

Fixed ground-based microwave radar and a moving object on a specific orbit together build the requirement for ISAR imaging geometry according to Figure 1 . The proper motion of the object generates the required synthetic aperture of length $L_{S A}$. Hence $R_{A}$ is the range at the beginning and $R_{E}$ at the end of the data acquisition process. A steerable antenna system provides capability of wide azimuth scanning required for high azimuth resolution. In parallel the integration time of total target observation is extended resulting in a useful enhancement of the Signal-to-Noise Ratio (SNR) in the radar image [2].

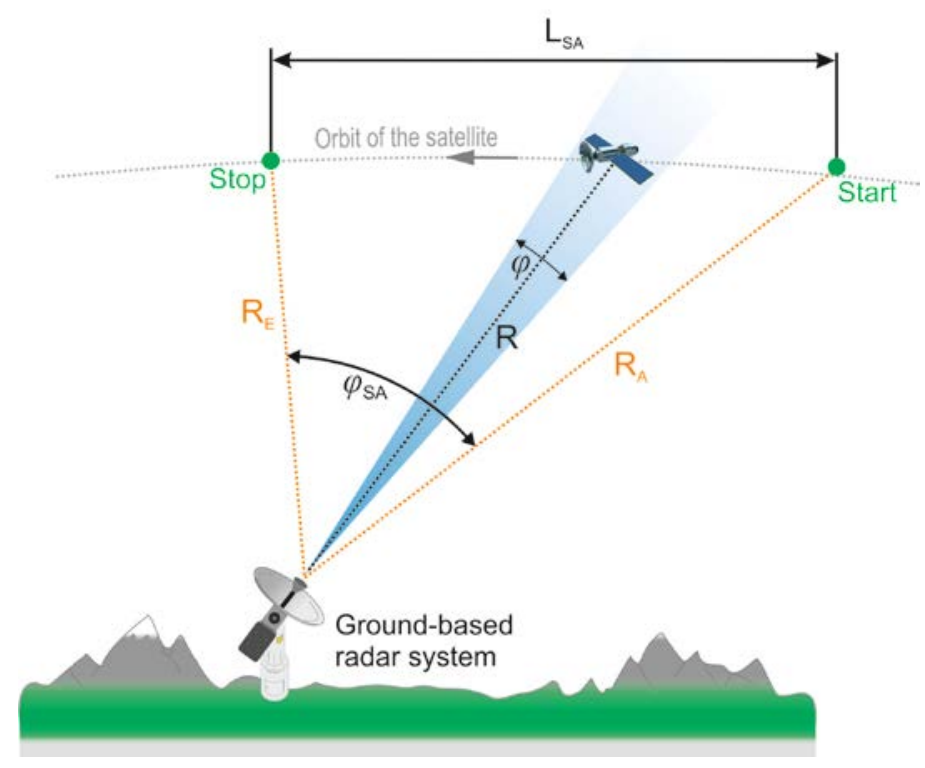

Figure 1.Operational principle for the imaging of spaceborne objects in LEO using ISAR techniques. For the length of the synthetic aperture between start and stop radar pulses are transmitted and received coherently in order to apply SAR processing for high-resolution images.

\subsection{Experimental hardware setup}

For the investigation of very high-resolution radar signatures of objects in space and, in order to assess requirements is spatial resolution, appropriate experiments using flexible and high-performance radar shall be carried out. In a first implementation the IoSiS system includes DLR multi-purpose advanced X-band radar system GigaRad in conjunction with a high power amplifier (HPA) and a Cassegrain antenna system of $9 \mathrm{~m}$ diameter for transmit (TX), and two directly fed dish antennas of $1 \mathrm{~m}$ diameter for receive (RX) as illustrated graphically in Figure 2 and pictorially in Figure 3. The two separated RX antennas may enable as well bi-static measurements in a different setup. The physical separation of TX and RX paths mainly provide sufficiently high cross-talk isolation considering the large bandwidth and the high pulse power of about $8 \mathrm{~kW}$. The $\mathrm{RX}$ antennas are rigidly installed on both sides of the main reflector in order to simplify parallel pointing directions for the observations. As feed system for the Cassegrain antenna type a broadband high-performance corrugated horn together with a sub-reflector were developed, ensuring spherical illumination of the main reflector at nearly frequency-independent aperture illumination within whole X band (Figure 3). In order to keep the distance between the HPA and the Cassegrain feed as short as possible for low transmission loss, the HPA is mounted on the antenna pedestal directly behind the main reflector in an airconditioned housing. Low loss electro-optical modulators/demodulators and optical fibers connect the GigaRad radar with the antenna system. These connections are used for the TX path and both RX paths as well as for calibration purposes, respectively. 
The main parts of the radar electronics, especially the digital subsystems, are located in a container nearby the antenna system. Here the whole IoSiS facility is configured and programmed for the observation of a specific object or satellite in LEO. Once configured and initialized, the antenna is automatically tracking the object based on TLE (True Line Elements) data. Figure 4 illustrates the various system parts of IoSiS outside and an inside look into the control rooms.

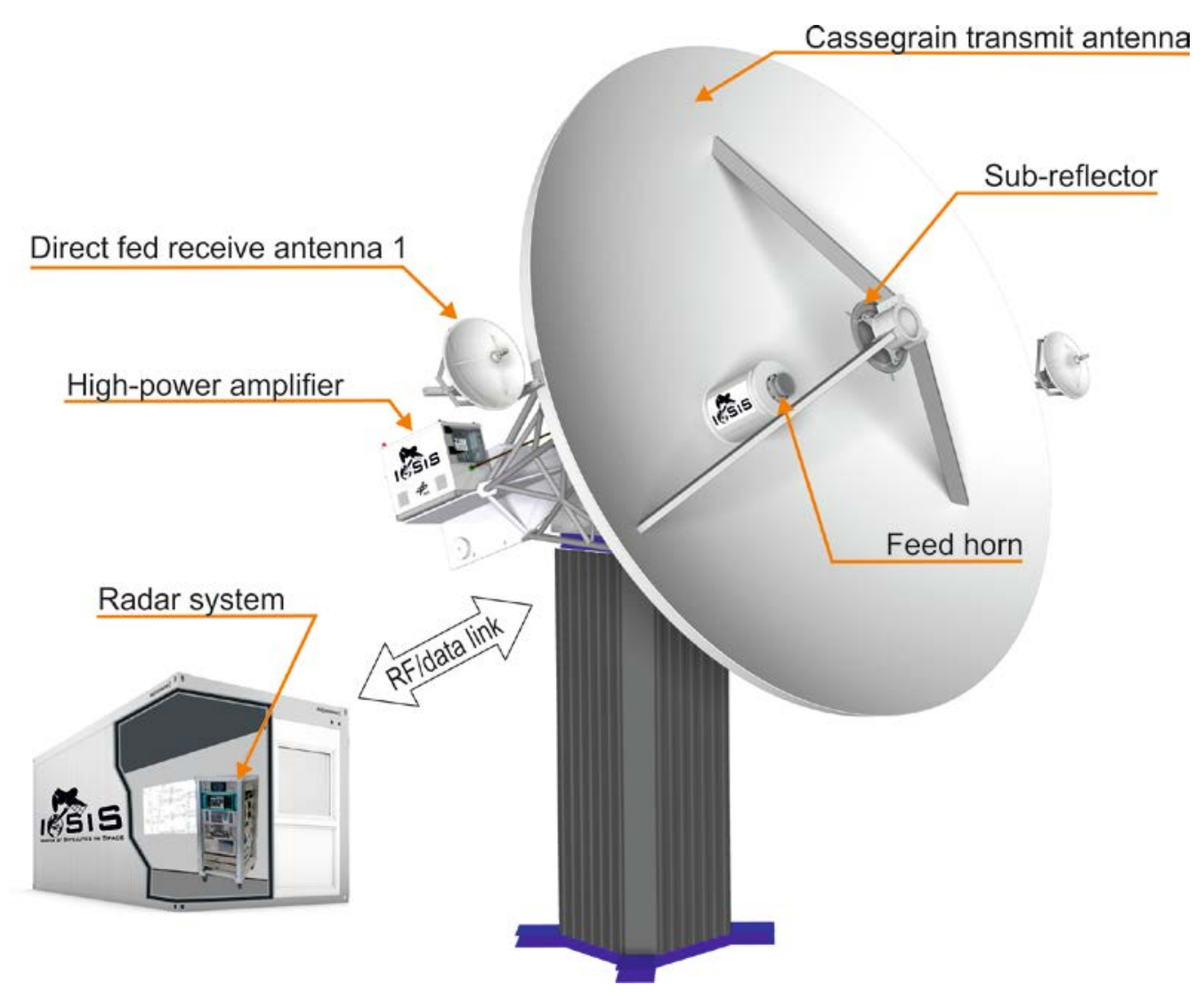

Figure 2 Sketch of main components of the IoSiS system as originally planned. 


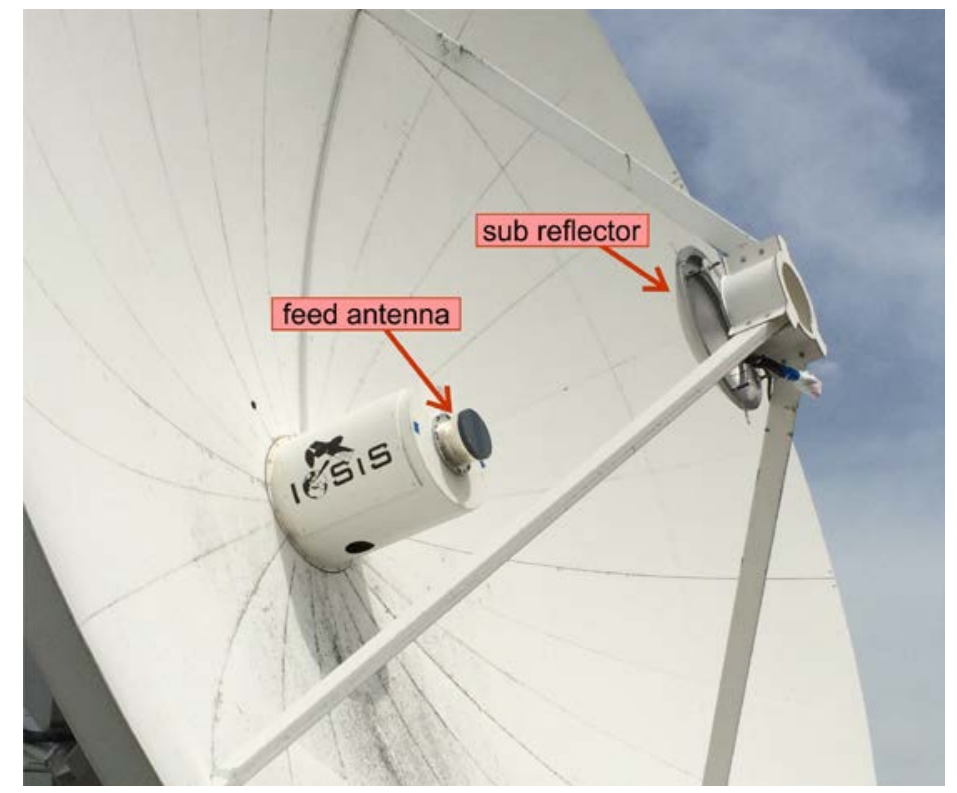

Figure 3 Photograph of the realized feed horn and sub-reflector of the overall Cassegrain antenna used for the TX radar subsystem.

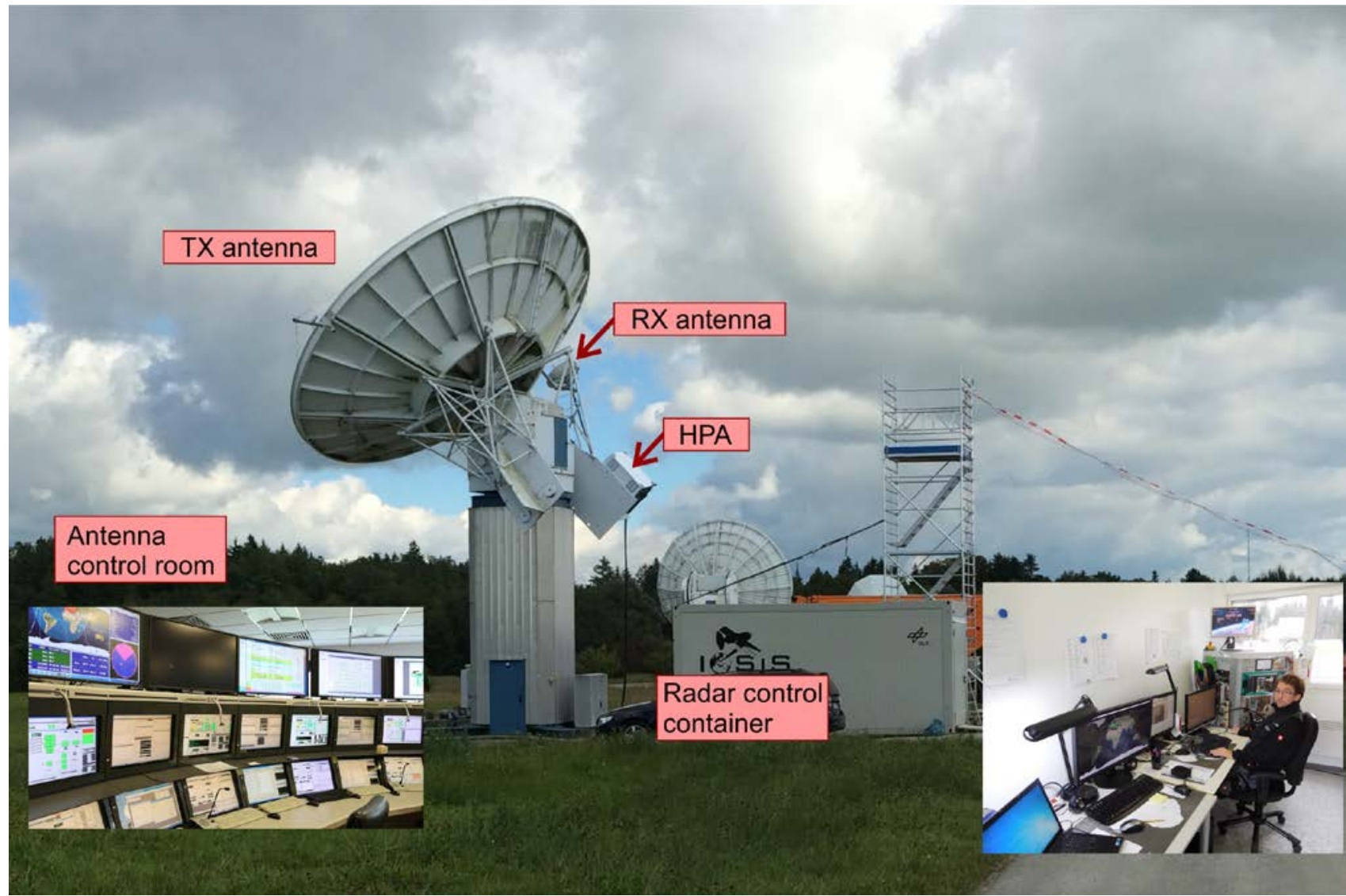

Figure 4. Photograph of the IoSiS system from outside showing the TX and RX antennas, the HPA installed in an airconditioned box and mounted on the antenna pedestal, and the container housing the GigaRad radar and the control terminals for whole system operation. The small photographs left and right show the inside of the antenna commanding room and the inside of the container. 


\subsection{Radar hardware description}

The high degree of flexibility of the GigaRad radar results in a very complex electronic design. For basic understanding a simplified block diagram is shown in Figure 5. The main functional parts of the instrument are:

- Arbitrary waveform generator (AWG),

- IQ (In-phase and Quadrature) transmit part,

- IQ receive part,

- High-speed data acquisition (HDA),

- Error correction network (ECN).

Purely digital signal generation is performed by using a high-performance arbitrary AWG, providing a maximum data rate of up to $10 \mathrm{GS} / \mathrm{s}$. This allows required flexibility on TX side in order to transmit arbitrary waveforms as well as an advanced error correction strategy. Both output signals of the AWG are coherently generated and fed to an IQ modulator. Then the signal is amplified, filtered, and transmitted. On the RX side the functional concept is similar, except the signal conditioning part before digitization using the HDA providing a sampling rate of $8 \mathrm{GS} / \mathrm{s}$. Hence, in order to fulfill the Nyquist criterion with some safety margins, the maximum analogue IF bandwidth is $3 \mathrm{GHz}$.

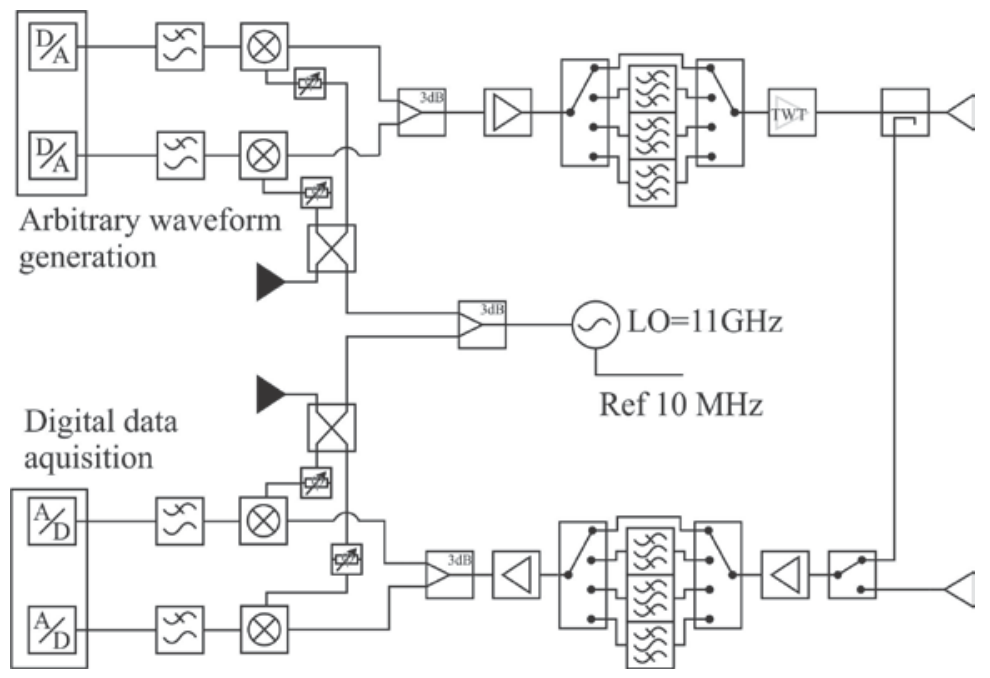

Figure 5. Simplified GigaRad block diagram.

In order to generate technically the maximum bandwidth as planned for IoSiS, the overall bandwidth is split in an upper sideband (USB) and a lower sideband (LSB) around the center frequency of $11 \mathrm{GHz}$. A frequency plan of the radar system is depicted in Figure 6. The maximum bandwidth of the radar system GigaRad is $6 \mathrm{GHz}$. The frequency limitation of the HPA reduces the bandwidth in the IoSiS configuration to a maximum of $4.4 \mathrm{GHz}$. This bandwidth can be either achieved by IQ modulation or notching unwanted image frequencies.

Especially the IQ circuit parts require proper error correction in order to achieve the desired image rejection as described in the next section. Another very important issue for very high-resolution SAR applications is the maintenance of sufficient coherence and therefore stability of the local oscillators. In case of GigaRad the implemented phase-locked source offers excellent low phase noise and spurious performance and can be additionally locked on an external ultrastable oscillator connecting both the digital and the frequency conversion sections. 


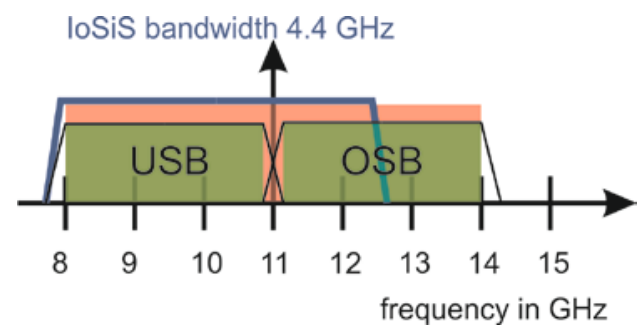

Figure 6. RF frequency plan of the GigaRad system capability (orange) and the band selection used for the IoSiS configuration (blue envelope).

A photograph of the basic radar hardware setup is shown in Figure. 7. The single units are arranged in a 19” rack. The highpower and low-noise amplification sections are excluded to allow a bi-static and high-power operation via optical transmission to a high-gain antenna system.

Due to the large complexity of the system, the different filter constellations, the error correction, and the required timing accuracy especially for the IoSiS mode, an Erasable Programmable Logic Device (EPLD) and a micro controller are used to control the whole instrument via appropriate clocks. Following the signal path of the block diagram in Figure 5 the original signal is generated in the AWG, followed by frequency conversion, and conditioning in the TX section. Then the signal is routed via an optical link to the extension modules shown here in the lower part of the rack, being located in the HPA box close to the antenna during IoSiS operation. Note that not only the RF signal has to be transmitted, but also the time sensitive control signals for duty cycle modulation of the HPA, and the receive gate switches being connected via a high-speed realtime bus. Figure 8 shows a block diagram of the electro-optical signal conversion for both TX and RX extension modules.

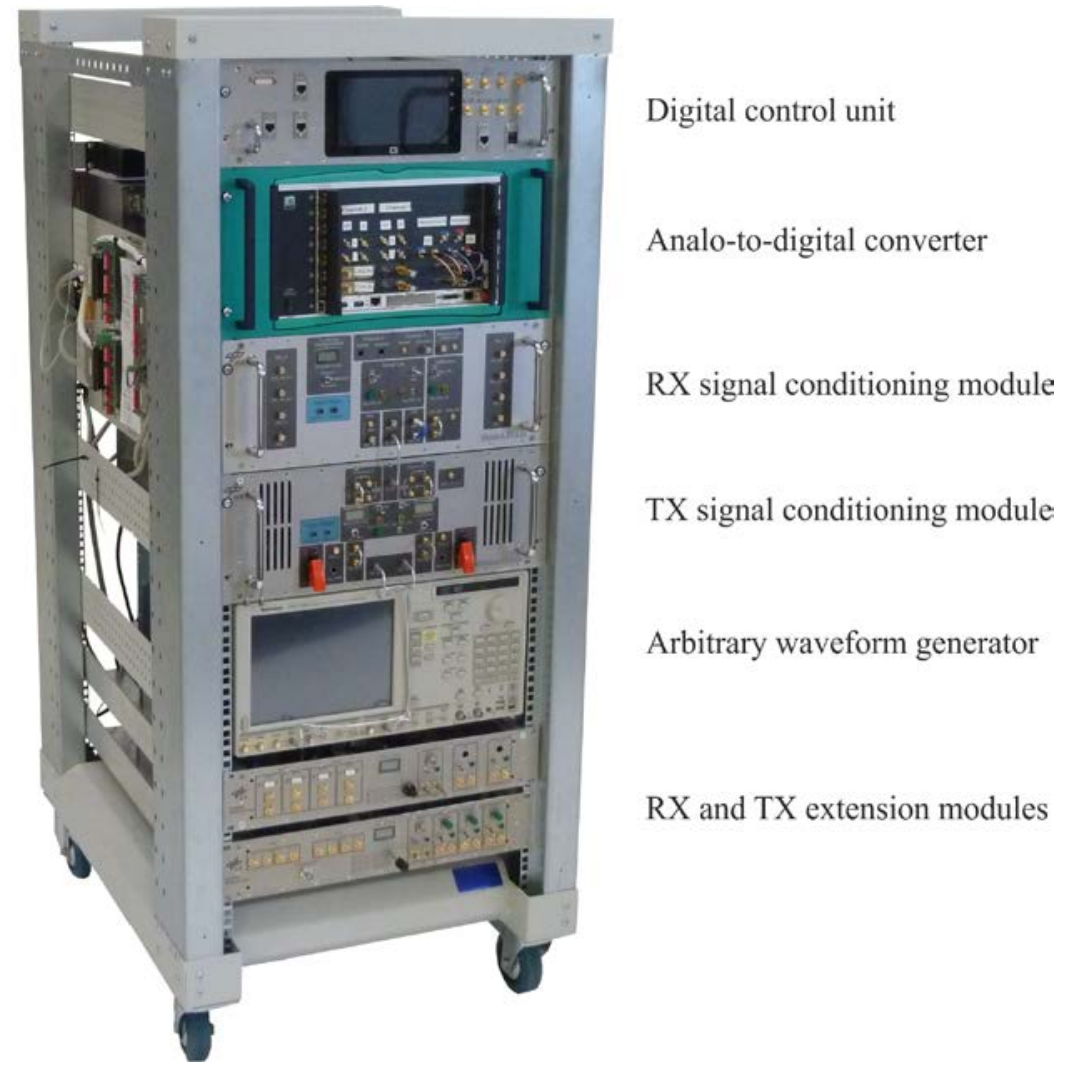

Figure 7. Photograph of the basic radar hardware setup of GigaRad including the extension modules, all being installed in a mobile 19"' rack. 


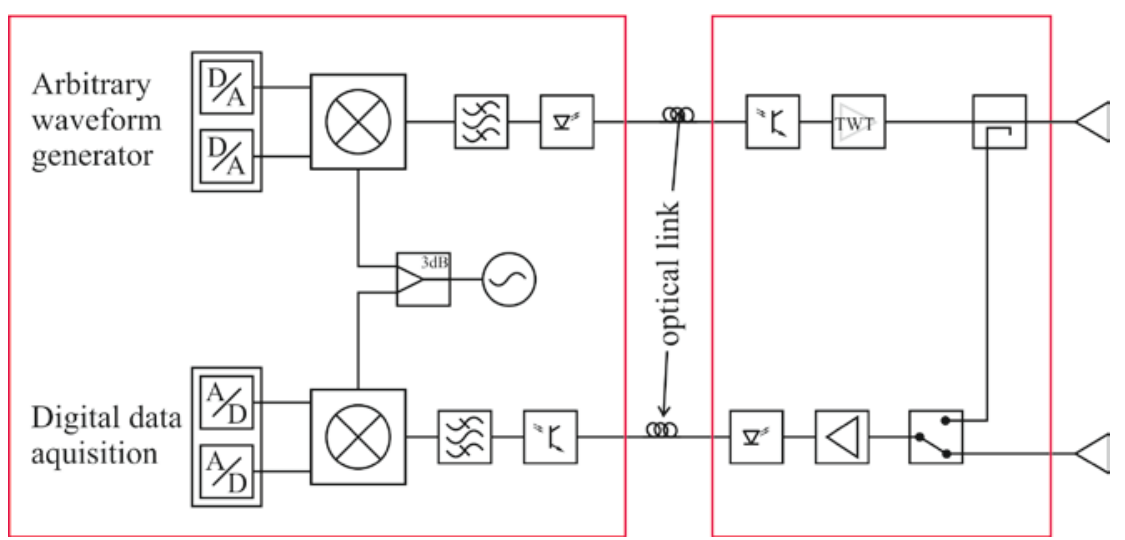

Figure 8. Simplified block diagram of the basic GigaRad instrument and the optically connected extension modules at the antennas.

\subsection{RF error correction and absolute calibration}

As mentioned earlier the proper rejection of image frequencies, error correction, and absolute calibration is necessary for high-quality images. Therefore in a first step the error correction for the IQ sections is performed. In Figure 9 the main strategy is illustrated. First of all the local oscillator signals are adjusted with manual phase trimmers to exactly $90^{\circ}$ phase difference at the mixer diodes of the TX and RX sections (path 1). This is the only step where external hardware is required, but this procedure has to be done only at longer-scale maintenance activities. The signal is analyzed at the left ends of the green paths in TX and RX sections prior the AWG and the HDA using an oscilloscope. The phase trimmers are adjusted accordingly.

In a second step I and Q paths are aligned separately using the different filters for the USB and LSB (path 2 and 3). Since the signal is sufficiently rejected in the unwanted frequency range by the filters, only one channel of the arbitrary waveform generator has to be used to compare the sampled signals in I and Q channels for amplitude and phase differences in the demodulation part. Here the signal is routed through the calibration path including the coupler right before the TX antenna and the switch following the RX antenna. A similar procedure is used for proper alignment of both AWG output channels.

In a third step the frequency response in the $\mathrm{TX}\left(\mathrm{H}_{\mathrm{TX}}\right)$ and $\mathrm{RX}\left(\mathrm{H}_{\mathrm{RX}}\right)$ chains is measured with respect to phase, group delay and amplitude (orange path) using the internal calibration path $\left(\mathrm{H}_{\mathrm{Cal}}\right)$. This transfer function in conjunction with the external calibration 4) determines the overall frequency response of the system. In this step especially the response of the antennas, the waveguides and the antenna feed systems are characterized in two transfer functions $\left(\mathrm{H}_{\mathrm{ANT} \_\mathrm{TX}}, \mathrm{H}_{\mathrm{ANT} \_\mathrm{RX}}\right)$. The external calibration is performed using a trihedral corner reflector having the transfer function $\left(\mathrm{H}_{\text {target }}\right)$. The latter is mounted on a crane in a known distance to the antennas providing therefor the known free-space transfer function $\left(\mathrm{H}_{\text {space }}\right)$. This setup is shown in Figure 10. 

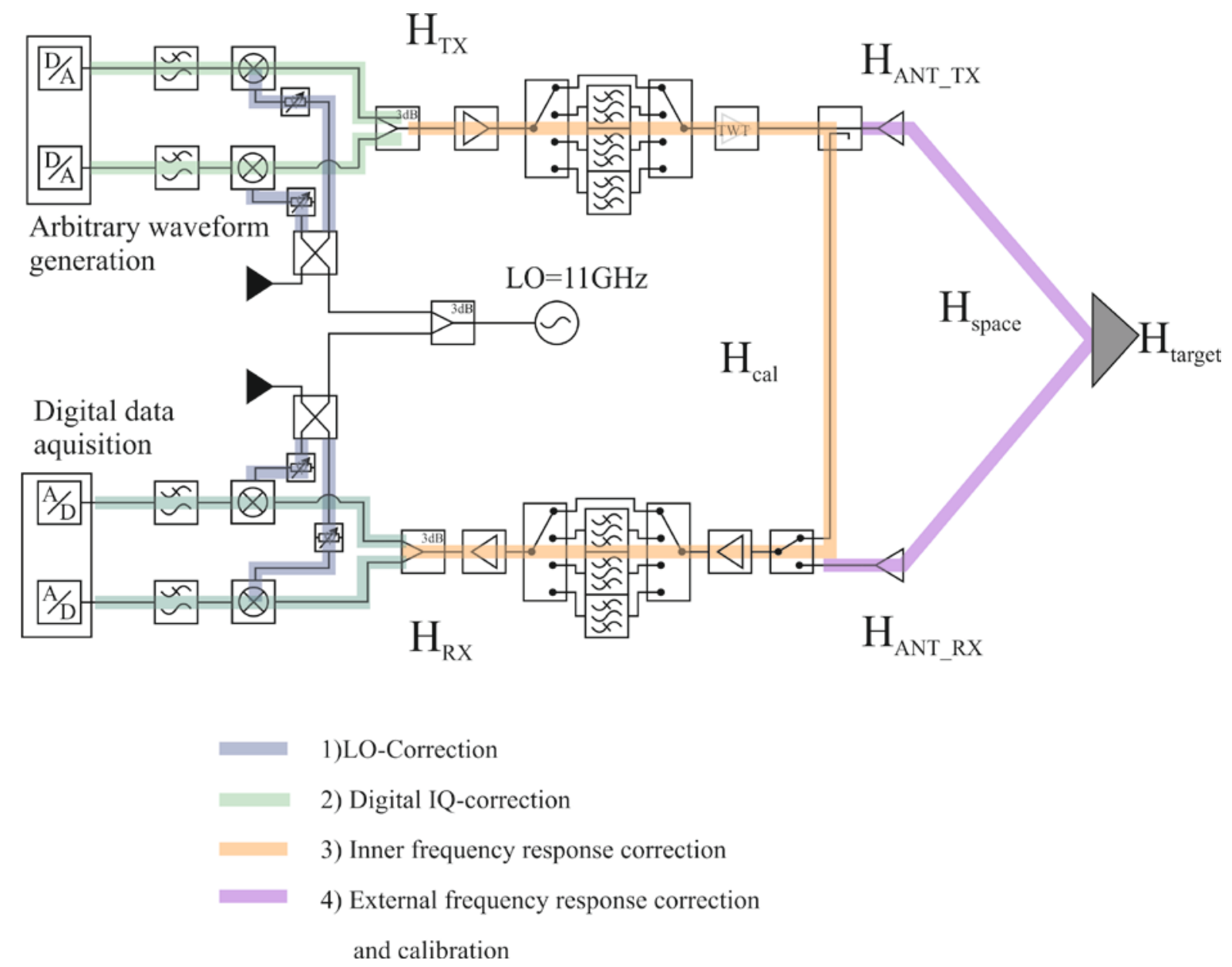

Figure 9. Block diagram explaining the system correction strategy 1): phase adjustment for the local oscillator signal in IQ modulation and demodulation, 2): amplitude and phase correction of the modulation/demodulation sections, 3): amplitude and phase correction of the RX and TX units, 4) amplitude and phase correction of the overall signal path including the antennas and waveguides.

Measurement 3) provides a correction function $\left(\mathrm{H}_{\text {corr_int }}\right)$ using the input signal $\mathrm{S}_{\text {chirp }}$ from the measured signal $\mathrm{S}_{\text {cal_int }}$ as formulated next:

$$
\begin{aligned}
& S_{c a l \_ \text {int }}=S_{c h i r p} H_{T X} H_{C A L} H_{R X} \\
& H_{T X} H_{R X}=\frac{S_{c a l_{\text {_int }}}}{S_{\text {chirp }} H_{C A L}}=H_{\text {corr_int }}^{-1}
\end{aligned}
$$

The absolute calibration in measurement 4) on one hand defines the relation to a physical unit, and on the other hand provides a correction for the antenna, feed systems and waveguides by the function $\left(\mathrm{H}_{\text {corr_ext }}\right)$ from the measured signal $\mathrm{S}_{\text {cal_corner. }}$.

$$
S_{\text {cal_corner }}=S_{\text {chirp }} H_{T X} H_{\text {ANT_TX }} H_{\text {space }} H_{\text {target }} H_{\text {ANT_RX }} H_{R X}
$$


By correcting the signal using the internal correction function, the external function can by computed using the knowledge of the transfer functions of the free-space and corner reflector.

$$
\begin{aligned}
& S_{\text {cal_corner_corr }}=S_{\text {cal_corner }} H_{\text {corr_int }}=S_{\text {chirp }} H_{\text {ANT_TX }} H_{\text {space }} H_{\text {target }} H_{\text {ANT_RX }} \\
& H_{\text {ANT_TX }} H_{\text {ANT_RX }}=\frac{S_{\text {cal_corner_corr }}}{S_{\text {chirp }} H_{\text {space }} H_{\text {target }}}=H_{\text {corr_ext }}^{-1}
\end{aligned}
$$

Knowing these two correction functions the measured data $S_{\text {data }}$ of an unknown target is corrected by multiplying with $\mathrm{H}_{\text {corr_int }}$ and $\mathrm{H}_{\text {corrext }}$. The correction of the actual free-space transfer function is part of a separate step in the overall IoSiS processor including appropriate atmospheric models. Since the internal calibration corrects also the non-stable, slowly time-varying parts of the radar instrument, this measurement is performed before and after each imaging procedure of a space object. The finally corrected responses now are given by

$$
\begin{aligned}
& S_{\text {data }}=S_{\text {chirp }} H_{T X} H_{\text {ANT_TX }} H_{\text {space }} H_{\text {unknown_target }} H_{\text {ANT_RX }} H_{R X} \\
& H_{\text {unknown_target }}=\frac{S_{\text {data }} H_{\text {corr_int }} H_{\text {corr_ext }}}{S_{\text {chirp }} H_{\text {space }}}
\end{aligned}
$$

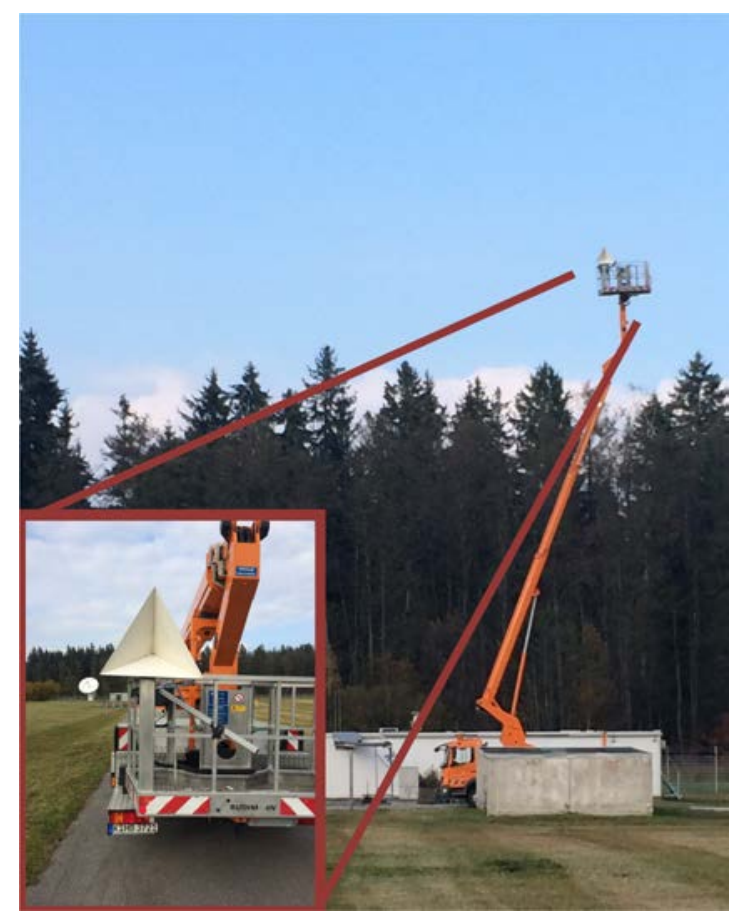

Figure 10. Photograph showing the setup for external calibration and frequency dependent error correction including antennas, feed systems and waveguides. A trihedral corner reflector in elevated position is used as well-known external radar target. 


\subsection{IoSiS measurement strategy}

Since the IoSiS instrument presently is in a commissioning phase the following steps for the imaging of a space-borne object are performed in a rather rudimentary way. For an operational system those steps are identical but executed more autonomously by full computer control.

1. Choice of a space object of interest.

2. Download of actual orbital parameters in terms of the TLEs.

3. Computation of the useful orbit segment of the space object as being visible from the geographical location of IoSiS.

4. Determination of the IoSiS timing parameters:

- range trajectory for the receive window,

- pulse repetition frequency (PRF),

- antenna tracking parameters.

5. Computation of the predicted pointing trajectory for the antenna control unit (ACU).

6. Initialization of the autonomous and GPS synchronized measurement process.

7. Measurement of range profiles of the space object for the predicted duration.

8. Application of the digital processing for error correction and performing final ISAR imaging.

The overall digital processing itself is still an ongoing development and not yet finished. This step is one of the most challenging parts of the IoSiS project due to the complexity of known and still unknown error sources and the demand for very high resolution in both range and azimuth directions.

\subsection{Pre-operational performance estimation}

For performance estimation prior final construction and operation of the IoSiS system a software tool was implemented, including relevant parts of the imaging system. Using that tool the hardware setup is optimized in parallel during development and construction. Figure 11 shows the corresponding relationships. The scene simulation is based on reflectivity maps gathered from three-dimensional object models [4]. The maps include triple, double, single and edge reflections. Those are computed only for the center frequency of the system in order to minimize computational load. The known orbit geometry based on TLE data together with the geographic position of the radar system determines the incidence angles and the maximum necessary range of azimuth angle for the synthetic aperture. Based on that adequate sensor parameters can be computed taking into account the pulse repetition frequency (PRF). Here the PRF together with the orbital height and object velocity determines the unambiguous range in azimuth direction. Subsequently the influence of the radar system including various error sources can be included [5] [1]. 


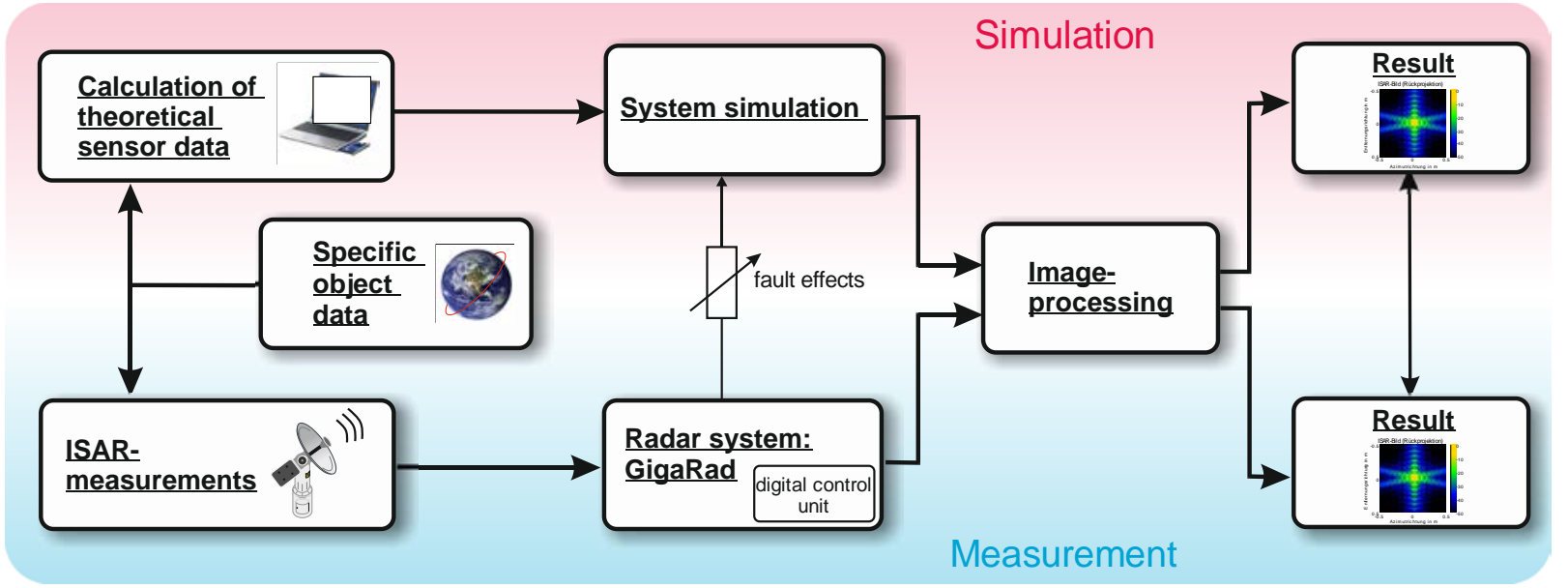

Figure 11. Structure of the IoSiS system simulator showing the relationships between pure system simulation and experimental verification of hardware. Independent of purely simulated or real data identical processing tools can be used for ISAR image generation.

Figure 12 illustrates the theoretical performance of the IoSiS system by a simulated ISAR image. As an appropriate target an Iridium communication satellite has been used as illustrated in the left drawing. The radar signature on the right was computed based on typical sensor parameters of IoSiS. The maximum bandwidth of $4.4 \mathrm{GHz}$ together with an azimuth integration angle of $24^{\circ}$ results theoretically in a quadratic resolution cell of approximately $3.5 \mathrm{~cm}$ width. This extremely high resolution allows precise determination of fine details of the satellite. For instance, all three aluminum antennas and the edges of the solar panels can be clearly identified. In addition, the high resolution provides nearly an optical appearance of the image showing the whole satellite structure even when part of the satellite cannot be seen due to shadowing. Comparing that image with a simulation of $7 \mathrm{~cm}$ resolution as shown in Figure 13 demonstrates clearly the enormous benefit of the higher resolution with respect to tiny details. Note that for these results the influence of possible error sources has not been considered here, but of course possible degradation of the image quality definitely is an issue. The simulation of such impacts is part of ongoing studies [5], [1].
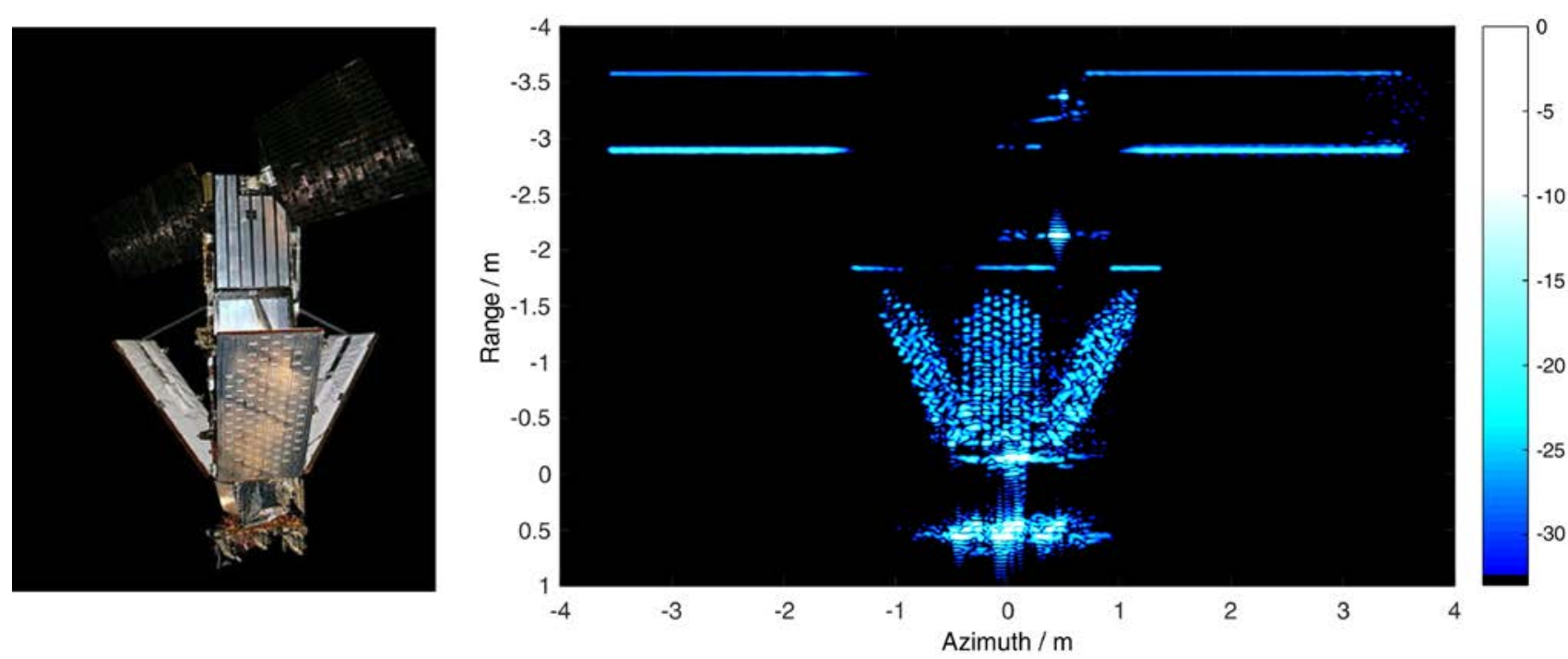

Figure 12: Drawing (left) and simulated ISAR image (right) of an Iridium communication satellite using a bandwidth of 4.4 $\mathrm{GHz}$ and an azimuth integration angle of $24^{\circ}$, being the width of the synthetic aperture. Resulting spatial resolution is $3.5 \mathrm{~cm}$. 


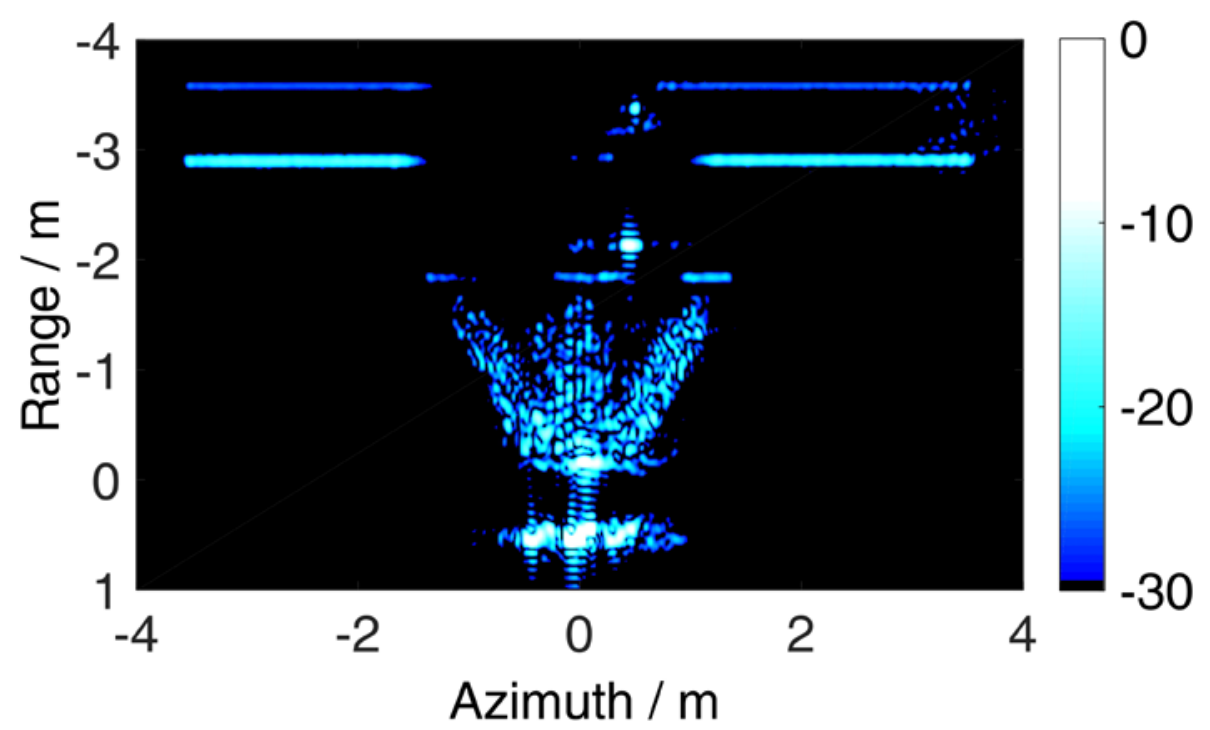

Figure 13. Simulated ISAR image of the identical satellite for a spatial resolution of $7 \mathrm{~cm}$ in range and azimuth.

\section{CONCLUSION}

The IoSiS system is considered to become a very high-performance imaging radar for space-borne objects in LEO. First simulation results give an idea about the image quality to be expected. In parallel to simulation work the hardware setup is developed and constructed. In this paper the experimental hardware configuration is outlined using a $9 \mathrm{~m}$ Cassegrain reflector antenna for TX and $1 \mathrm{~m}$ direct fed dish for RX. The high-power TX amplification and the low-noise RX section are installed on the backside of the TX antenna. As the high-performance key radar system the innovative multi-purpose instrument GigaRad is used and adapted to the intended application. The very high spatial resolution and the application of the system require an intensive error correction methodology and absolute calibration as shown in this paper. The possible correction of expected atmospheric delays and the compensation of inaccuracies in the knowledge of target location are part of analysis using the simulator. Based on such knowledge the ISAR processor is adapted and optimized successively in order to produce the demanded high-resolution radar images of objects like satellites in space.

\section{ACKNOWLEDGMENT}

We are thankful to our colleges of DLR satellite ground station in Weilheim, Germany, giving us the opportunity and lots of support installing our instrument on their $9 \mathrm{~m}$ antenna. Especially we like to thank Martin Häusler and Amanuel Geda for their endorsement and technical support.

We are also grateful to our technicians Harald Schreiber and Frank Reinwaldt for support in development and construction of the tremendous digital and software part of the radar. Without suitable instrument control and highquality PCB boards the instrument would never work.

We are also immensely grateful to our mechanical workshop guided by Peter Heitzer. This team provides us with all necessary high-precision mechanics and they are helping us to install the instrument.

\section{REFERENCES}

[1] Anger, S, Jirousek, M., Peichl, M. “GigaRad - a versatile high-resolution ground-based pulse radar for advanced remote sensing research”, Proc. EUSAR, Berlin, Germany, 2014.

[2] Mensa, D.: “High Resolution Radar Cross-Section Imaging”, Artech House, 1981 
[3] Jirousek, M., Iff, S., Anger, S., Peichl, M., “GigaRad - a multi-purpose high-resolution ground-based radar system concept, error correction strategies and performance verification”. International Journal of Microwave and Wireless Technologies, 7(3/4), pp 443-451. Cambidge University Press. (2015)

[4] Anglberger, H., Speck, H., Suess, H., "Application of Simulation Techniques for high resolution SAR Systems", IEEE International Geoscience and Remote Sensing Symposium (IGARSS), Munich, 2012.

[5] Anger, S., Peichl, M., Dill, S., Jirousek, M., Schreiber, E., "IoSiS - A high-performance imaging radar for surveillance of objects in low earth orbit", Proceedings of European Conference of Synthetic Aperture Radar (EUSAR), Hamburg, Germany, 2016. 